OPEN ACCESS

\section{Clustering and turbulence modulation in particle laden shear flows}

To cite this article: P Gualtieri et al 2011 J. Phys.: Conf. Ser. 333012007

View the article online for updates and enhancements.

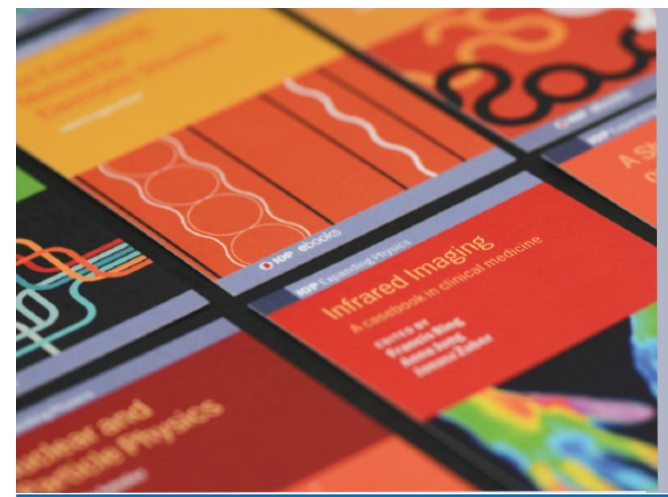

\section{IOP ebooks}

Bringing together innovative digital publishing with leading authors from the global scientific community. Start exploring the collection-download the first chapter of every title for free. 


\title{
Clustering and turbulence modulation in particle laden shear flows
}

\author{
P. Gualtieri, F. Picano, G. Sardina \& C.M. Casciola \\ Dipartimento di Ingegneria Meccanica e Aerospaziale, Università di Roma La Sapienza, Via \\ Eudossiana 18, 00184 Roma Italy \\ E-mail: paolo.gualtieri@uniroma1.it
}

\begin{abstract}
Turbulent fluctuations induce the commonplace phenomenology on the transport of small inertial particles known as clustering. Particles spread disuniformly and form aggregates where their local concentration is much higher than it is in nearby rarefaction regions, the voids, where in extreme cases not even a single particle can be found. The underlying physics has been exhaustively analyzed in statistically homogeneous and isotropic flows under the so called oneway coupling regime, i.e. in conditions where the momentum exchange between the carrier fluid and the disperse phase is negligible. Recently it has been shown that the addition of a mean flow might have dramatic effects on the disperse phase, i.e. the mean flow, through its large scale anisotropy, induces a preferential orientation of the clusters. Due to inertial effects, their directionality can even increase in the smallest scales, contrary to the expectation based on the isotropy recovery behavior of velocity fluctuations. This finding opens new issues in presence of large mass loads, when the momentum exchange between the two phases becomes significant and the back-reaction of the particles on the carrier flow cannot be neglected. These aspects are discussed here by addressing direct numerical simulation data of particle laden homogeneous shear flow in the two-way coupling regime. Consistently with previous findings we observe an overall depletion of turbulent fluctuations. In particular, particles with order Kolmogorov scale relaxation time induce the energy depletion of the classical inertial scales and the amplitude increase of the smallest ones where the particle back-reaction pumps energy into the turbulent eddies increasing their energy content. We find that increased mass loads result in the substantial broadening of the energy co-spectrum thereby extending the range of scales driven by anisotropic production mechanisms. This is due to the clusters which form the spatial support of the back-reaction field and give rise to a highly anisotropic forcing active down to the smallest scales.
\end{abstract}

\section{Introduction}

Transport of inertial particles is involved in several fields of science such as droplets growth and collisions in clouds $[1,2]$, the plankton accumulation in the oceans $[3,4]$ or the plume formation in the atmosphere [5]. At the same time multiphase flows are the base of several technological applications. The inertial particles dynamics is crucial in designing injection systems of internal combustion engines [6], to prevent sediment accumulation in pipelines $[7,8]$ or for the appropriate dimensioning of several industrial devices [9].

The relevant physical aspect in particles dynamics consists in their finite inertia which prevents them from following the fluid trajectories. Most evident is the "preferential accumulation" which, in inhomogeneous flows such as wall bounded flows, occurs in the form of the so called "turbophoresis", i.e. preferential localization of particles in the near wall region 
[10, 11]. An exhaustive review of the subject can be found e.g. in [12], see also [13] and the recent paper [14] for a physical explanation in terms of statistical properties of velocity fluctuations in the near wall region.

When the idealized conditions of isotropic turbulence are addressed, preferential accumulation manifests itself in the form of small scale clustering. The disperse phase forms small scale aggregates where most particles concentrate, separated by void regions of small particle density, see e.g. [15] and references therein.

So far the effect of turbulent transport on particle dynamics has been studied extensively in many flow configurations. Much less is known about the effect the disperse phase may have on the carrier flow demanding for a renewed effort in this direction see e.g. [16, 17]. It is expected that, under proper coupling conditions, the momentum exchange between the two phases might become relevant in driving the turbulent fluctuations away from their universal equilibrium state predicted by Kolmogorov in the early forties. Clearly, in contrast to the one-way coupling regime, addressing these effects calls into play the more realistic two-way coupling mechanism, where the disperse phase provides an active modulation of velocity fluctuations.

In this context, many authors first studied the simplest flow configuration, i.e. decaying homogeneous isotropic turbulence $[18,19,20,21,22]$, by addressing the decay rate of turbulence intensity and the modification of the energy spectra under two-way coupling regime. Other numerical investigations analyzed the statistically steady state of isotropic turbulence [23] showing a non uniform distortion of the energy spectra, i.e. a damping of turbulent fluctuations at large scales and a relative enhancement at the smallest ones. In some cases, the overall effect of back reaction on the carrier phase is reported as an attenuation of turbulence fluctuations controlled by the mass load ratio defined as the ratio of total disperse phase mass to fluid mass, see e.g. $[24,25]$. Similar effects have also been observed for particles larger than the Kolmogorov scale showing that the crossover wavenumber characterizing the transition between dumped and enhanced energy modes is related to the particles finite size [26]. Other important issues such as the increase of the particles settling velocity under gravity have been addressed both numerically [27] and experimentally [28]. By breaking isotropy gravity leads to a preferential augmentation of turbulence intensities along the vertical direction while in the transverse directions augmentation is only observed below the Taylor scale. Other interesting effects of the momentum exchange between the carrier fluid and the disperse phase is observed in the context of grid generated spatially decaying turbulence. In fact, starting from an isotropic state the feedback of the particles seems to lead to an anisotropic flow as turbulent fluctuations develop downstream $[29,30]$. An extensive review of the back-reaction effects on isotropic turbulence might be found in Ref. [31]. Concerning wall bounded flows many authors considered the classical geometry of the channel $[32,33,34]$ or of the pipe flow $[35,36]$. Due to inhomogeneity and anisotropy new features emerge such as the preferential suppression of turbulence intensities in the wall normal direction [34], the enhancement of large scale anisotropy as measured by velocity variances [33] or even the occurrence of drag reduction [32]. A substantial modification of turbulent kinetic energy production has been reported in Ref. [37] in the context of a time evolving homogeneous shear flow.

In any case, as isotropy is broken by the presence of gravity or by the mean streamwise advection, the back-reaction of the disperse phase seems to immediately originate strong anisotropies in the carrier phase. Motivated by recent findings in the context of anisotropic clustering [38, 39], we consider here the modulation of turbulence by transported particles addressing the particle laden homogeneous shear flow in the two-way coupling regime. Such flow can be considered as a sort of bridge between the idealized conditions of isotropic turbulence and the more realistic geometries of wall bounded flows, since it preserves spatial homogeneity and retains the anisotropy of shear flows.

It is worth recalling that, under shear, turbulent fluctuations are strongly anisotropic at 
the largest scales due to production of turbulent kinetic energy via interaction of the mean velocity gradient and turbulent fluctuations. At smaller scales, below the so-called shear scale $L_{S}=\sqrt{\epsilon / S^{3}}$-where $\epsilon$ is the energy dissipation rate and $S$ the mean shear-inertial energy transfer usually prevails. In such conditions re-isotropization of turbulent fluctuation takes place following a route described in [40, 41, 42]. However, turbulent fluctuations in the oneway coupling regime are found to induce an anisotropic clustering of the disperse phase which persists down to the smallest scales [38]. Actually, in contrast to the small scale behavior of velocity fluctuations, particles aggregates do not lose their directionality. Their anisotropy even increases down to the viscous scales where clusters still keep memory of the spatial orientation of the large scale coherent motions.

Here we consider the same flow under the two-way coupling scenario. In this more complex case the physical result we achieve consists in a view of multi-phase turbulent flows consistent with the multiscale forcing addressed in [43]. Small scales anisotropic clusters acts as a source/sink of momentum for the turbulent motions distributed all along the range of scale. They deplete the energy from the largest inertial scales which is in part retrieved in the lowinertial/dissipative range. Here the clusters keep the velocity fluctuations to a higher excitation state than expected on the basis of the standard Kolmogorov theory. As we will see such back energy scatter is highly anisotropic in shear flow, hence the small scales fluid motions are prevented from recovering isotropy and eventually increment their anisotropy.

\section{Methodology}

Concerning the carrier fluid, the velocity field $\mathbf{v}$ is decomposed into a mean flow $\mathbf{U}=S x_{2} \mathbf{e}_{1}$ and a fluctuation $\mathbf{u}$ where $\mathbf{e}_{1}$ is the unit vector in the streamwise direction, $x_{2}$ denote the coordinate in the direction of the mean shear $S$ and $x_{3}$ is in the spanwise direction. Rogallo's technique [44] is employed to rewrite the Navier-Stokes equations for velocity fluctuations in a deforming coordinate system convected by the mean flow according to the transformation of variables $\xi_{1}=x_{1}-S t x_{2} ; \quad \xi_{2}=x_{2} ; \quad \xi_{3}=x_{3} ; \quad \tau=t$. The resulting system

$$
\nabla \cdot \mathbf{u}=0 ; \quad \frac{\partial \mathbf{u}}{\partial \tau}=(\mathbf{u} \times \boldsymbol{\zeta})-\nabla \pi+\nu \nabla^{2} \mathbf{u}-S u_{2} \mathbf{e}_{1}+\mathbf{F},
$$

is numerically integrated by a pseudo-spectral method combined with a fourth order RungeKutta scheme for temporal evolution. In equations (1) $\boldsymbol{\zeta}$ is the curl of $\mathbf{u}, \pi$ is the modified pressure which includes the fluctuating kinetic energy $|\mathbf{u}|^{2} / 2, \nu$ is the kinematic viscosity and $\mathbf{F}$ denote the back-reaction due to the disperse phase. The latter consists of diluted particles with mass density $\rho_{p}$ much larger than the carrier fluid $\rho_{f}$. The approximation of point particles can be adopted whenever the particle diameter $d_{p}$ is much smaller than the typical turbulence scales. It follows that the only relevant force is the Stokes drag [45]. Accordingly, the equations for particles position $x_{i}^{p}(t)$ and velocity $v_{i}^{p}(t)$ read

$$
\frac{d x_{i}^{p}}{d t}=v_{i}^{p} ; \quad \frac{d v_{i}^{p}}{d t}=\frac{1}{\tau_{p}}\left[v_{i}\left(x^{p}, t\right)-v_{i}^{p}(t)\right],
$$

where $v_{i}\left(x^{p}, t\right)$ is the instantaneous fluid velocity evaluated at $x_{i}^{p}(t)$ and $\tau_{p}=\rho_{p} d_{p}^{2} /\left(18 \nu \rho_{f}\right)$ is the Stokes relaxation time. Particles' velocities are decomposed as $v_{i}^{p}=U_{i}\left[x_{k}^{p}(t)\right]+u_{i}^{p}$ where $u_{i}^{p}$ denotes the particle velocity deviation with respect to the local mean flow of the carrier fluid. Finally by using Rogallo's transformation eqs. (2) can be written as

$$
\frac{d \xi_{i}^{p}}{d \tau}=u_{i}^{p}-S \tau u_{2}^{p} \delta_{i 1} ; \quad \frac{d u_{i}^{p}}{d \tau}=f_{i}^{p} .
$$

Equations (1) and (3) are integrated in computational space. In fact, the computational box gets distorted in physical space by the mean flow advection. The re-meshing procedure is 
periodically applied to allow long time integrations. By exploiting periodicity in the $\xi_{1}$ direction, the computational domain is transformed back into a non skewed domain every $\Delta t_{r}=2 / S$, preventing the unbounded growth of the wavenumbers, see [46] for further details on the numerics adopted. In equation (3) $f_{i}^{p}=\tau_{p}^{-1}\left[u_{i}\left(\xi^{p}, \tau\right)-u_{i}^{p}(\tau)\right]-S u_{2}^{p} \delta_{i 1}$ is the expression of the Stokes drag acting on the $p^{\text {th }}$ particle. In the so-called two way coupling regime an equal and opposite force acts on the carrier fluid accounting for the momentum exchange between the two phases.

Modeling the back reaction in numerical simulations is an issue [47]. The local distortion of turbulence due to the disperse phase can be captured only resolving the boundary of each particle on the computational grid. In the so-called resolved particle simulations several approaches have been proposed ranging from finite volume schemes [48] to Lattice Boltzman Methods [49]. However other approaches are possible once it has been recognized that the flow close to a small particle can be locally approximated as a Stokes Flow. In Ref. [50] the Stokes solution is used to provide appropriate boundary conditions to the Navier-Stokes equations close to each particle. Clearly these approaches, even though they provide the exact momentum coupling between the carrier and the disperse phase, are feasible only for a relatively small number of particles. When particles are much smaller than the turbulent scales they can be considered as material points i.e. as point source/sinks of momentum for the carrier fluid. Within this approximation several other methodologies are available. For instance, in the force coupling method [51] the disturbance flow produced by the particles is modeled as a regularized steady Stokes solution corresponding to a force monopole and quadrupole while the singular steady Stokes solution is employed in Ref. [34]. In our case we adopt the particle in cell method introduced in Ref. [52] where the particle is regarded as a point source of momentum for the carrier fluid. This is the simplest approach to model the two way coupling regime widely adopted in the literature even if the methodology might suffer of lack of numerical convergence in estimating the interphase momentum transfer [53]. Accordingly, the resulting force on the fluid is computed as

$$
\mathbf{F}=-\frac{N_{c}}{N_{p}} \Phi \sum_{p}^{n(\xi)} \mathbf{f}^{p}
$$

where the sum is extended to all the $n(\xi)$ particles belonging to the computational cell centered at point $\xi$. In eq. (4) $N_{c}$ denote the number of Eulerian cells, $N_{p}$ is the total number of particles and $\Phi$ denote the mass load ratio i.e. the ratio between the mass of the disperse phase $M_{p}=N_{p} \pi \rho_{p} d_{p}^{3} / 6$ and the carrier fluid $M_{f}=\rho_{f} V_{f}$ where $V_{f}$ is the volume of the computational box. Fluid properties are known in an Eulerian frame while particles evolve along their own Lagrangian trajectories requiring a first interpolation when the fluid velocity is computed at the particle position, namely $u_{i}\left(\xi^{p}, \tau\right)$. An other interpolation is required when the back-reaction on the fluid is computed since $f_{i}^{p}$ is known at the particle position. In fact, the force acting on the $p^{t h}$ particle is re-distributed via inverse interpolation to the nearest Eulerian grid points where the fluid velocity is defined. Equations (3) are integrated by the same fourth order Runge-Kutta scheme used for the Navier-Stokes equations and the interpolation adopt a tri-linear scheme.

The data we analyze come from a statistically steady Direct Numerical Simulation (DNS) of a homogeneous shear flow. The mean shear induces velocity fluctuations which are strongly anisotropic at the larger scales driven by production while, at smaller separations, the classical energy transfer mechanisms become effective in inducing re-isotropization. The position of the shear scale $L_{S}$ was found crucial to determine whether small scale isotropy recovery eventually occurs. For these reasons $L_{S}$ plays an important physical role in shear turbulence where it enters the two basic control parameters $[54,55]$. The first one is the Corrsin parameter, $S_{c}^{*}=$ $S(\nu / \epsilon)^{1 / 2}=\left(\eta / L_{S}\right)^{2 / 3}$, where $\eta=\left(\nu^{3} / \epsilon\right)^{1 / 4}$ is the Kolmogorov scale. The Corrsin parameter can be recast in terms of the Taylor-Reynolds number $1 / S_{c}^{*} \propto \operatorname{Re}_{\lambda}=\sqrt{5 /(\nu \epsilon)}\left\langle u_{\alpha} u_{\alpha}\right\rangle$, where 
$u_{\alpha}$ is the $\alpha^{\text {th }}$ Cartesian component of the velocity fluctuation and the angular brackets denote ensemble averaging. The second parameter is the shear strength $S^{*}=S\left\langle u_{\alpha} u_{\alpha}\right\rangle / \epsilon=\left(L_{0} / L_{S}\right)^{2 / 3}$, with $L_{0}=\left\langle u_{\alpha} u_{\alpha}\right\rangle^{3 / 2} / \epsilon$ the integral scale of the flow. The former determines the extension of the range of scales below the shear scale and above the Kolmogorov length $\eta$. When this range is sufficiently extended, i.e. the Taylor-Reynolds number is large enough, small scale isotropy recovery is likely to occur in the fluid velocity field. The latter, instead, fixes the range of scales directly affected by the geometry of the forcing. This is the anisotropic range between integral and shear scale.

The Navier-Stokes equations are integrated in a $4 \pi \times 2 \pi \times 2 \pi$ periodic box see table 1 for a full description of the dataset. The Kolmogorov scale is $\eta=0.07$ which corresponds to $K_{\max } \eta$ ranging from 5.8 to 15.5. Note the very accurate resolution of the smallest scales required to have smooth fields in view of accurate interpolations and re-distribution of the back-reaction on the carrier fluid. Accurate resolution of the smallest scales is also crucial when addressing the statistical properties of the viscous dissipation field [56, 57].

Concerning the disperse phase, the dynamics is controlled by the ratio of the particles relaxation time $\tau_{p}$ to a characteristic flow time scale, typically the Kolmogorov time scale $\tau_{\eta}=(\nu / \epsilon)^{1 / 2}$, i.e. the relevant control parameter is the Stokes number $S t_{\eta}=\tau_{p} / \tau_{\eta}$. When the two-way coupling regime is considered other non dimensional parameters are required to describe the momentum exchange between the two phases, namely the density ratio $\rho_{p} / \rho_{f}-$ assumed to be much larger than unity- and the mass load fraction $\Phi=M_{p} / M_{f}$. In fact, the mass load can be expressed as $\Phi=\left(\rho_{p} / \rho_{f}\right) \Phi_{v}$ where $\Phi_{v}=V_{p} / V_{f}$ is the volume fraction. When $\rho_{p} / \rho_{f} \gg 1$, the mass load $\Phi$ can be order one when the volume fraction is still very small, order $10^{-3}$ in the present case. In such conditions inter-particles collisions might be safely neglected see e.g. [16] while the momentum coupling between the two phases must be retained. For the simulations summarized in table 1 particles are injected in an already fully developed turbulent flow. Their position is initialized at random homogeneous points with initial velocity matching the fluid velocity at particle position. The total number of particles is changed to achieve different values of the mass load parameter while the Stokes number is kept constant. To this purpose, after discarding an initial transient, 150 statistically independent snapshots are used to compute the relevant statistical observables. The snapshots are collected at each remesh time

\begin{tabular}{cccc} 
run & $N_{x} \times N_{y} \times N_{z}$ & $\Phi$ & $N_{p}$ \\
\hline$a$ & $192 \times 96 \times 96$ & - & - \\
$b$ & $384 \times 192 \times 192$ & 0.2 & 327500 \\
$c$ & $384 \times 192 \times 192$ & 0.4 & 655000 \\
$d$ & $384 \times 192 \times 192$ & 0.8 & 1310000 \\
$d_{l}$ & $192 \times 96 \times 96$ & 0.8 & 1310000 \\
$d_{h}$ & $512 \times 256 \times 256$ & 0.8 & 1310000
\end{tabular}

Table 1. Direct Numerical Simulation dataset. Navier-Stokes equations are integrated in a $4 \pi \times 2 \pi \times 2 \pi$ periodic box with a resolution of $N_{x} \times N_{y} \times N_{z}$ Fourier modes. The $3 / 2$ dealiasing rule is adopted to compute the non linear terms. The Taylor Reynolds number is $R e_{\lambda}=50$ and the shear parameter is $S^{*}=7 . N_{p}$ is the number of particles whose Stokes number based on the Kolmogorov time is $S t_{\eta} \simeq 1$ with $\tau_{p}=0.4$. The mass load parameter (see text for definition) is denoted by $\Phi, \rho_{p} / \rho_{f}=2000$ indicate the density ratio between the disperse and carrier phase. In our case $d_{p} / \eta \simeq 0.1$. The first four entries of the table provide for the effect of increasing the mass load ratio for a given grid resolution, runs $a-d$. Comparison of run $d_{l}$ and $d_{h}$ with the reference case $d$ provide the sensitivity to grid resolution for a given mass load ratio. 
when the computational box comes back to its unskewed configuration.

\section{Particles Clustering}

A visual impression of instantaneous particles' configurations is provided in figure 1 where slices of the domain in selected coordinate planes are displayed for different values of the mass load parameter $\Phi$ at $S t_{\eta}=1.05$. Hereafter the Stokes number is defined with respect the Kolmogorov time of the simulation operated in the one-way coupling regime. In fact, as we will see in $\S 4$, the back-reaction leads to an overall depletion of the viscous energy dissipation rate and consequently to an alteration of the Kolmogorov time-scale $\tau_{\eta}=(\nu / \epsilon)^{1 / 2}$. In all the four cases
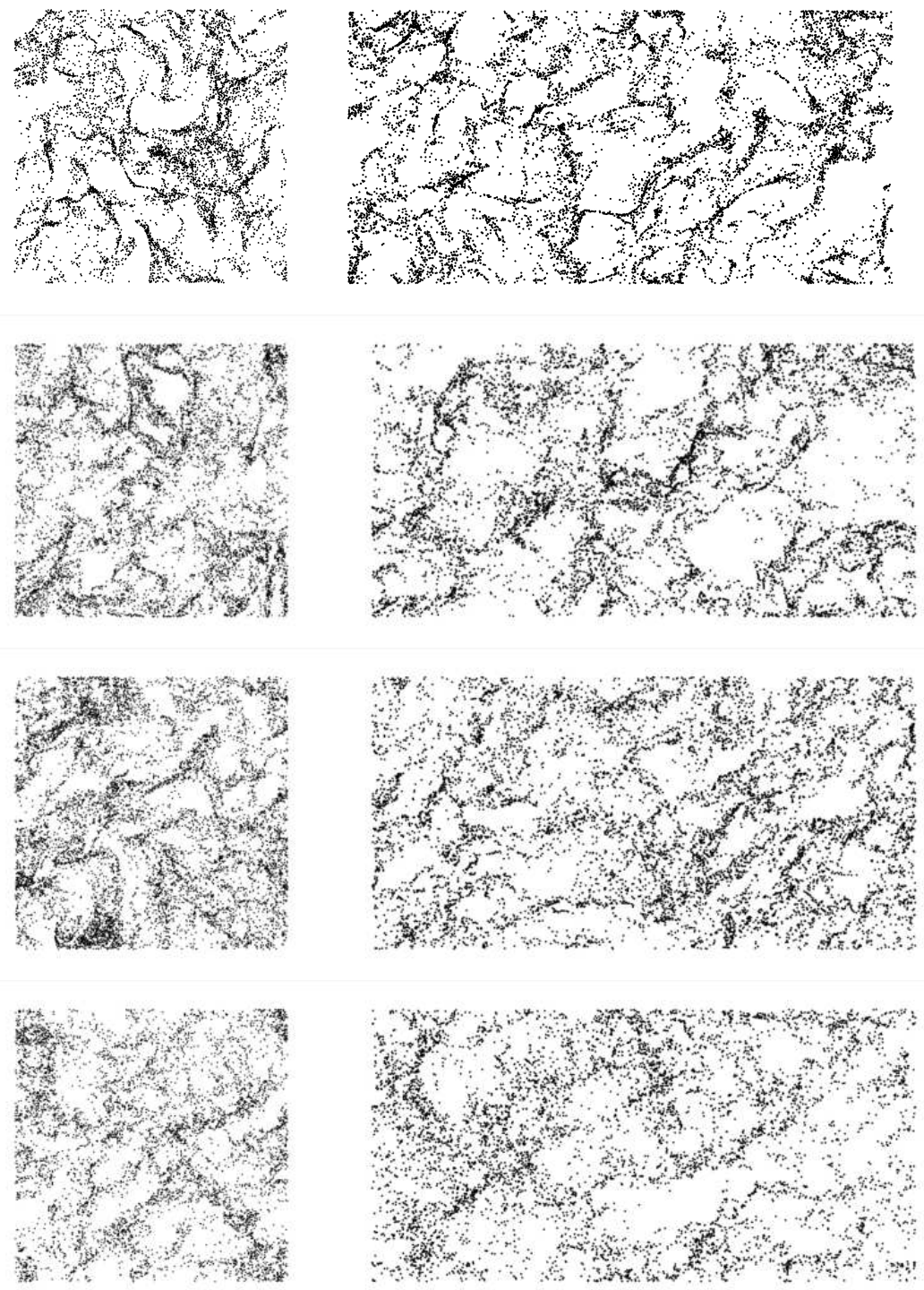

Figure 1. Snapshots of particle positions for increasing values of the mass load parameter $\Phi=0,0.2,0.4,0.8$ from top to bottom. For all the datasets $S t_{\eta}=1$. Left column thin slice in the $y-z$ plane; right column slice in the $x-y$ plane. The slice thickness is of the order of a few Kolmogorov scales. 
reported in figure 1, the disperse phase is characterized by a multi-scale distribution of particles concentration and voids. From the figure the shear induced orientation of the clusters is apparent even though the concentration of the particles in the accumulation regions seems to be reduced as the coupling effects become relevant. At a first sight this effect might be explained in terms of an alteration of the Kolmogorov time scale resulting in a smaller effective Stokes number i.e. for a given relaxation time $-\tau_{p}=0.4$ in our case-particles might be expected to behave more like tracers in the two-way coupling regime. However, a more quantitative analysis shows that this is not the case. The modification of the Kolmogorov time-scale, even though the energy dissipation rate is significantly changed at high mass loads, is small and corresponds to an alteration of the Stokes number which can not explain the observed depletion of the clustering intensity in the two-way coupling regime. For instance, the Stokes number ranges from $S t_{\eta}=1.05$ when turbulence is not modified by the particles to $S t_{\eta}=0.90$ in the most severe coupling conditions at $\Phi=0.8$. In conclusion, the observed depletion of the clustering intensity calls into play non trivial physical effects which are more likely related to an overall alteration turbulent fluctuations rather than an oversimplified explanation in terms of an alteration of the Stokes number.

In this paper we limit ourselves to the description of anisotropic clustering under the two-way coupling conditions by considering the Angular Distribution Functions (ADF) introduced in [38]. Essentially the ADF measures the number of particles' pairs at separation $r$ in the direction $\hat{\mathbf{r}}$ and is defined as

$$
g(r, \hat{\mathbf{r}})=\frac{1}{r^{2}} \frac{d \nu_{r}}{d r} \frac{1}{n_{0}},
$$

where $n_{0}=0.5 N_{p}\left(N_{p}-1\right) / V_{f}$ is the volume density of particles' pairs and $\nu_{r}(r, \hat{\mathbf{r}}) d \Omega$ is the numbers of particles' pairs contained in the spherical cone of radius $r$ with axis along $\hat{\mathbf{r}}$ and amplitude $d \Omega$. The spherical average of the $\mathrm{ADF} g_{00}(r)=1 /(4 \pi) \int_{\Omega} g(r, \hat{\mathbf{r}}) d \Omega$ is called the Radial Distribution Function (RDF) and has been already used to characterize particles clustering in isotropic conditions. The ADF extend the tool to anisotropic conditions retaining information on the directionality of the clusters. The behavior of the RDF near the origin $g_{00}(r) \propto r^{-\alpha}$

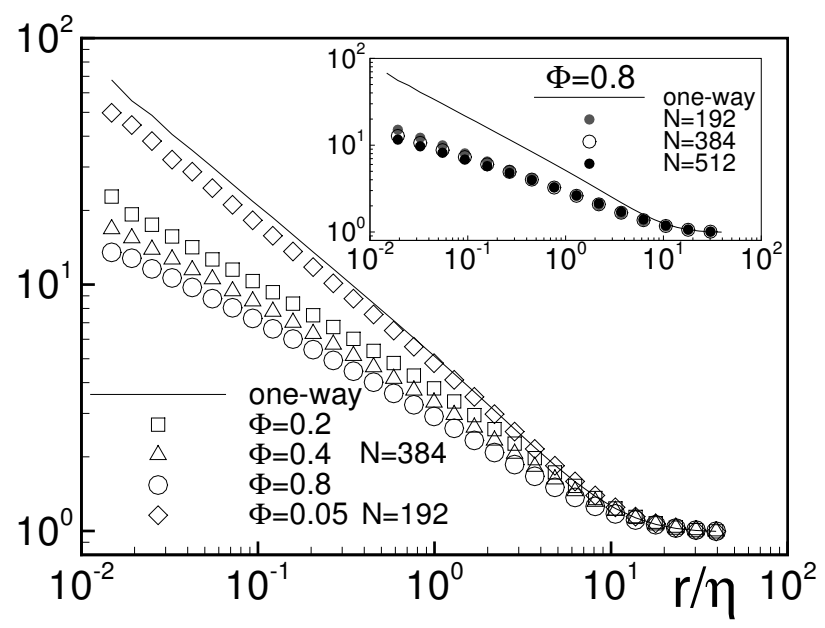

Figure 2. Projection of the ADF in the isotropic sector $g_{00}$ as a function of separation. The main panel address the effect of an increasing mass load for a given resolution of the carrier phase. The inset provides sensitivity of the data to the grid resolution for the highest mass load $\Phi=0.8$. Error-bars (not shown) are of the order of the symbols size in the entire range of scales. 
can be shown to be related to important geometrical features of the clusters. In particular $\mathcal{D}_{2}=3-\alpha$ is the so-called correlation dimension [58] of the multi-fractal measure associated with the particle density. A positive $\alpha$ indicates the occurrence of small scale clustering.

The RDF is shown in figure 2 for the different values of the mass load parameter $\Phi$. The data show the quantitative alteration of the clustering intensity. In fact, the back-reaction progressively attenuates the particles concentration at small scales leading to a smaller value of the scaling exponent $\alpha$. The modification is substantial. As the mass load is progressively increased the number of particles pairs at small scales is reduced about seven times as inferred by comparing the small scale values of $g_{00}$ obtained in the one-way coupling simulation (solid line) against the two-way coupling data at $\Phi=0.8$ (open circles) in figure 2 . The plot shows the smooth convergence of the two-way coupled simulation to the uncoupled case for very small values of the mass load, see for instance the data at $\Phi=0.05$ in comparison with those at $\Phi=0$. Concerning statistical accuracy, in the case of the isotropic projection of the ADF shown in figure 2 the error-bars (not shown) are smaller than the symbol size. In conclusion, the behavior confirms the qualitative impressions gained by simple inspection of the instantaneous particles patterns reported in figure 1 where the clusters are definitely less defined in the case $\Phi=0.8$ than at $\Phi=0$, compare bottom and top panels of the figure, respectively. In any case clustering, though partially attenuated, is a persistent feature of the two-way coupling regime as measured by the exponent $\alpha$. In order to exclude any bias in our results due to the numerics, in the inset of figure 2 we address the RDF in the most severe coupling conditions. For the highest mass load $\Phi=0.8$ we have performed two additional simulations with a coarser and finer resolution with respect to the reference case of run $d$, namely run $d_{l}$ and $d_{h}$ of table 1 respectively. The data confirm that the attenuation of the clustering is a genuine physical effect resulting from the momentum exchange between the carrier and disperse phase.

In view of turbulence modification, the anisotropy of particles' clusters will play a crucial role to be discussed in the next section where the analysis of the back-reaction effects on the carrier

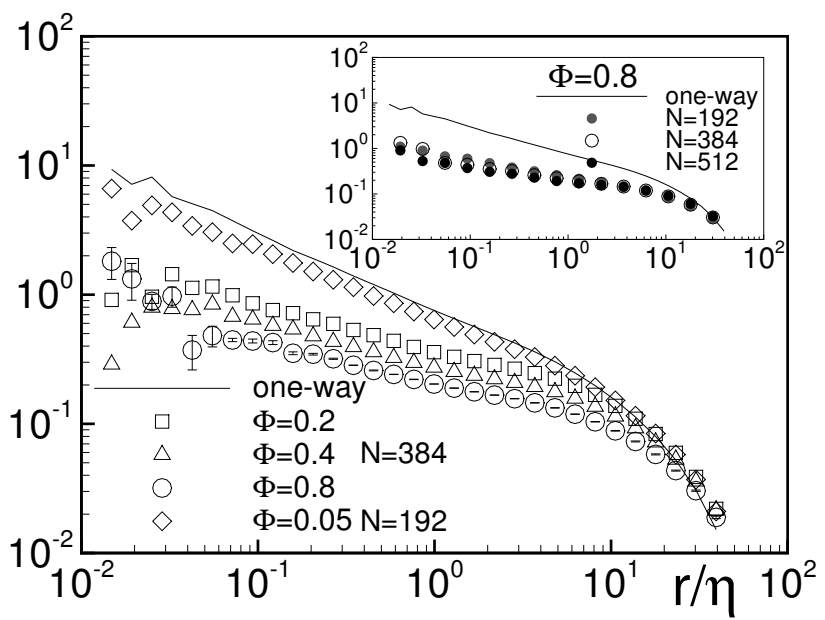

Figure 3. Projection of the ADF in the most energetic anisotropic sector $g_{2,-2}$ as a function of separation. The main panel addresses the effect of the mass load for a given resolution of the carrier phase. The inset provides the sensitivity to the grid resolution for the highest mass load $\Phi=0.8$. Error-bars for the data at $\Phi=0.05,0.2,0.4$ (not shown) are of the same order of those reported for the most severe coupling case at $\Phi=0.8$. 
fluid is addressed. For this reason, we complete the analysis of clustering under two-way coupling regime by characterizing the anisotropy of the aggregates. This can be done by exploiting the directionality properties of the ADF. Its angular dependence $g(r, \hat{\mathbf{r}})$ can be resolved in terms of spherical harmonics

$$
g(r, \hat{\mathbf{r}})=\sum_{j=0}^{\infty} \sum_{m=-j}^{j} g_{j m}(r) Y_{j m}(\hat{\mathbf{r}}),
$$

achieving a systematic description both in terms of separation $r$, accounted for by the coefficients $g_{j m}(r)$, and in terms of directions, described by the shape of the basis functions $Y_{j m}(\hat{\mathbf{r}})$. Each successive subspace, here labeled $j$, accounts for increasing levels of anisotropy consistently with the geometrical meaning of the spherical harmonics. This tool, introduced by [59], allowed for the systematic study of small scale isotropy recovery, see for instance $[60,61,62,40]$ and the exhaustive review in [63].

In figure 3 we show the most energetic anisotropic projection of the ADF namely $g_{2-2}$. As in the one-way coupling regime, by reducing the scale separation the intensity of the anisotropic projection increases indicating directionality in the particles aggregates. As for the RDF, the small scale behavior of $g_{2-2}$ is controlled by the mass load ratio while the numerical resolution seems to play a negligible role, see the right panel of figure 3 where $g_{2-2}$ is shown for $\Phi=0.8$ and different resolutions. The statistical convergence of this observable might be an issue since it samples only few preferential directions. The error-bars on the data for the worse case at $\Phi=0.8$ are always smaller than the symbol size up to $r / \eta=0.1$ below which the error becomes appreciable on the scale of the plot. To correctly infer about the persistency of directionality at small scales, in figure 4 we show the most energetic anisotropic projection normalized by the RDF, namely the ratio $g_{2-2} / g_{00}$. This indicator quantifies the average level of anisotropy of particles' clusters at that particular scale. We observe that the back-reaction on the carrier fluid partially reduces the small scale anisotropy of the disperse phase when compared with

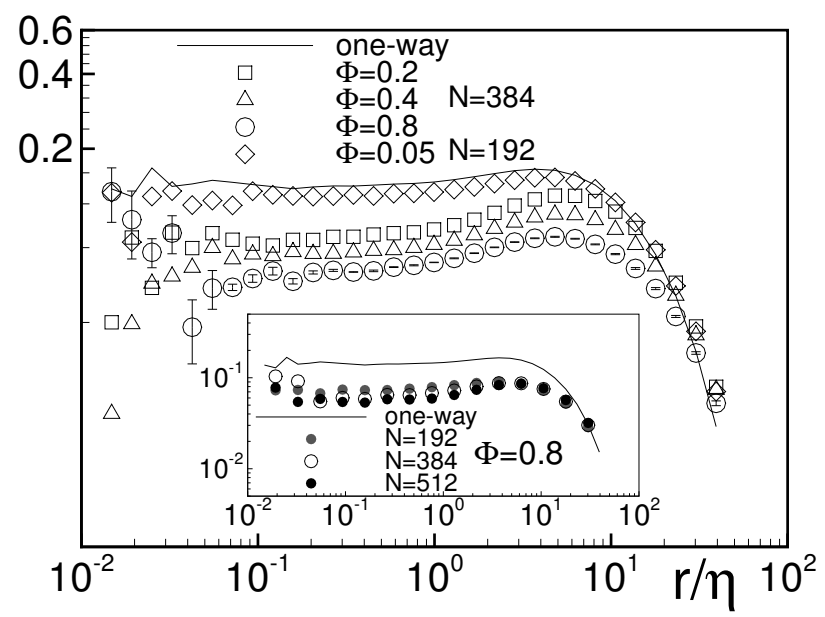

Figure 4. Normalized anisotropic projection of the ADF $g_{2-2} / g_{00}$ as a function of separation. The left panel addresses the effect of the mass load for a given resolution of the carrier phase. The right panel show the sensitivity to the grid resolution for the highest mass load $\Phi=0.8$ The error-bars for the data at $\Phi=0.05,0.2,0.4$ (not shown) are of the same order of those reported for the most severe coupling case at $\Phi=0.8$. 
the one-way coupling regime. In contrast to the saturation observed under one-way coupling conditions (solid line), the ratio $g_{2-2} / g_{00}$ seems to slightly decrease to eventually saturate to a lower value when the smallest scales are approached. In any case, going down the scale range, the anisotropy of the clusters is still present also in the two-way coupling regime. The overall behavior is not substantially altered from what we already described in previous papers [38] on the one-way coupling regime denoting a persistency of the clusters' directionality up to the smallest viscous scales.

\section{Turbulence Modulation}

In presence of two-way coupling, the support of the particle reaction field on the fluid is mainly given by the sets where most of the disperse phase concentrate. Actually clusters are organized in multi-scale sets whose orientation is controlled by the large scales anisotropy [38]. The effect is strong and the particles never achieve small scale isotropy, despite the Richardson cascade, in absence of back reaction, drives the fluid velocity fluctuations towards an isotropic state. We infer that the fluid is excited by a highly anisotropic, spectrally non-compact forcing, quite an unusual circumstance in turbulence. In these conditions, the back-reaction of the disperse phase is expected to deeply alter the structure of turbulence. Energy extraction/injection is now strongly anisotropic and active from the largest down to the smallest scales of the flow leaving no opportunity for a classical inertial range.

We start our description of turbulence modulation observing that, in the homogeneous shear flow, the mean velocity profile is imposed both in the one-way and the two-way coupling regimes. This excludes the modification of the mean flow itself as it could happen instead in channels or jet flows. Any alteration of turbulence can be directly ascribed to an intrinsic distortion of velocity fluctuations due to the inter-phase momentum exchange. The simplest quantities which
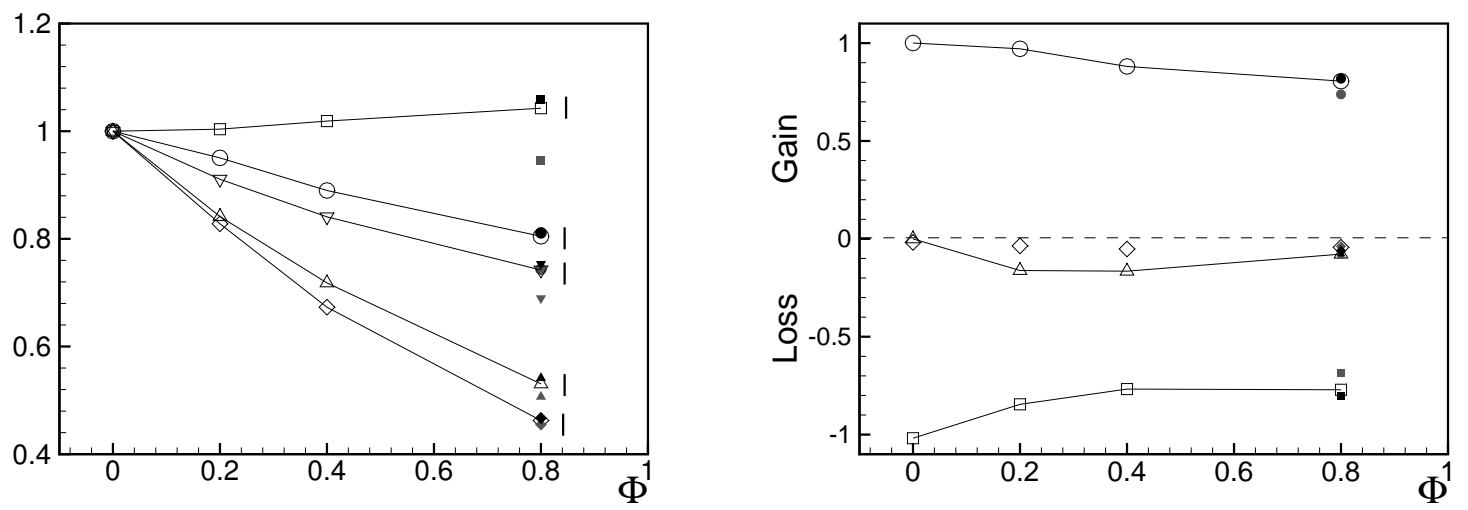

Figure 5. Left: effect of the mass load $\Phi$ on the turbulent fluctuations. Data normalized with the corresponding values of the un-coupled case. Open symbols refers to simulations $a-d$ in table 1. Filled symbols refers to cases $d_{l}$ (grey) and $d_{h}$ (black). $\left\langle u_{1}^{2}\right\rangle$ (square), $\left\langle u_{2}^{2}\right\rangle$ (delta), $\left\langle u_{3}^{2}\right\rangle$ (diamond), $1 / 2\left\langle u_{i} u_{i}\right\rangle$ (gradient), $-\left\langle u_{1} u_{2}\right\rangle$ (circle). The vertical tick of amplitude 0.04 provides the statistical error of the data. Right: turbulent kinetic energy budget. Data normalized with the production rate of the un-coupled case $\mathcal{P}_{0}$. Production $\mathcal{P}$ (circles), viscous energy dissipation $\epsilon$ (squares), energy flux intercepted by particles $\epsilon_{p}$ (delta). The algebraic sum $\mathcal{P}-\epsilon-\epsilon_{p}$ is also reported (diamonds) to check the quality of the budget. Filled symbols refers to cases $d_{l}$ (grey) and $d_{h}$ (black). Error-bars are of the order of the symbol size. 
characterize the response of turbulence are single point statistics such as the turbulent kinetic energy, the energy dissipation rate and, as always for shear flows, the Reynolds shear stress. The turbulent kinetic energy is addressed in figure 5 as a function of the mass load ratio. In addition, in the same figure, data at fixed mass load $\Phi=0.8$ and different grid resolutions, namely run $d_{l}$ and $d_{h}$, are reported to check the grid sensitivity. Consistently with previous findings in the context of isotropic turbulence and wall bounded shear flows, by increasing the mass load ratio the turbulent fluctuations are found to be progressively attenuated.

The directionality of the mean flow leads to a significant difference in the three normalized velocity variances. The streamwise component $\left\langle u_{1}^{2}\right\rangle$-squares in figure 5 -is the least affected by the back-reaction. Nonetheless the trend is toward its slight increase in comparison with the uncoupled case. The other two components, $\left\langle u_{2}^{2}\right\rangle$ (delta) and $\left\langle u_{3}^{2}\right\rangle$ (diamonds), are progressively attenuated as the coupling becomes more relevant. The depletion-up to $50 \%$ at $\Phi=0.8-$ overwhelms the slightly increasing trend of the streamwise variance leading to an overall reduction of turbulent kinetic energy. The resolution check for case $\Phi=0.8$ (gray and black filled squares in the figure) confirms the quality of the results. The impression is that the back-reaction enhances the large scale anisotropy of the flow. The concurrent depletion of the Reynolds shear stress is noteworthy, since it drags along the reduction of turbulent kinetic energy production.

The steady state turbulent kinetic energy balance in the two-way coupling regime, $\mathcal{P}=\epsilon+\epsilon_{p}$, involves a new term, the energy exchanged with the particles through the Stokes drag $\epsilon_{p}=$ $\langle\mathbf{F} \cdot \mathbf{u}\rangle$, whereas the production term $\mathcal{P}_{0}=-S\left\langle u_{1} u_{2}\right\rangle_{0}$ simply balances the viscous dissipation $\epsilon_{0}$ in the uncoupled case (the subscript stands for $\Phi=0$, no back-reaction). Actually at steady state the average particle kinetic energy is constant implying $\epsilon_{p}=\langle\mathbf{F} \cdot \mathbf{u}\rangle>0$ since $\left\langle\mathbf{F} \cdot \mathbf{v}_{\mathbf{p}}\right\rangle=0$. Hence the energy provided by the Reynolds stress work is partially intercepted by the backreaction field which represents an alternative dissipation channel, see $[64,65,66]$ for a similar mechanism in the context of polymer laden flows and $[67,68]$ for more recent work on the
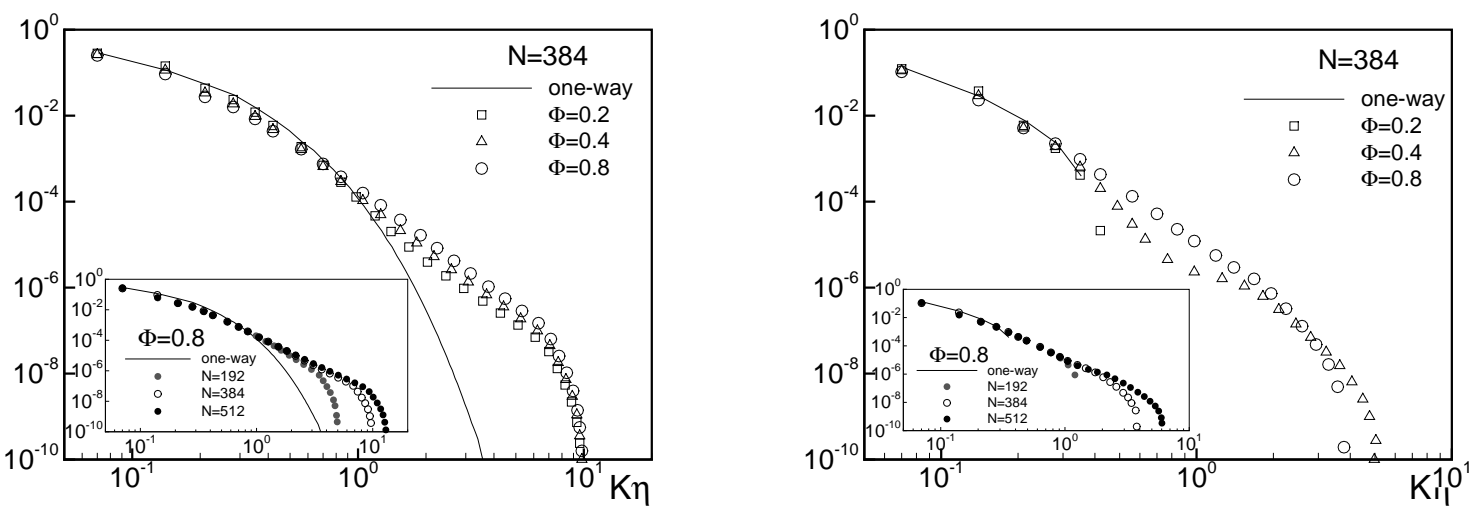

Figure 6. Effects of the mass load on the energy spectrum (left) and cospectrum (right) for a fixed spectral resolution of the carrier phase. Data of the one-way coupled simulation run $a$ (solid line) are compared with two-way coupled simulations operated for incresing mass loads $\Phi=0.2,0.4,0.8$ (symbols) which correspond to run $b$ (square), $c$ (delta) and $d$ (circle) respectively, see table 1 . In the inset effect of the grid resolution at the fixed mass load ratio $\Phi=0.8$. Data of the one-way coupled simulation (solid line, run $a$ in table 1) are compared against two-way coupled data at $\Phi=0.8$ obtained with different resolutions of the carrier phase. Symbols correspond to runs $d_{l}, d$ and $d_{h}$ of table 1 . 
subject. The energy budget is addressed in graphical form in figure 5 . The striking effect of the coupling is the considerable reduction of the production rate $\mathcal{P}-20 \%$ at $\Phi=0.8$-which entails the reduction of the viscous dissipation $\epsilon$. Although typically smaller than $\epsilon$, intriguing is also the behavior of the Stokes dissipation $\epsilon_{p}$ (deltas in figure 5) which achieves its maximum-order $15 \%$-at intermediate mass loads.

A more detailed analysis of turbulence modulation calls into play the spectral distribution of turbulent kinetic energy. In the spectra shown in figure 6 , as $\Phi$ increases, turbulent fluctuations are first attenuated in an intermediate range of scales to be eventually enhanced at small scales before being shut down by viscosity. In other words, the Stokes drag intercepts energy from the classical cascade at intermediate scales where velocity fluctuations are damped. The intercepted energy is partly pumped back to the fluid at small scales increasing the energy content by orders of magnitude, compare solid line (one-way coupling) and open symbols (two-way coupling) in figure 6 . The overall effect is a reduction of turbulent kinetic energy, essentially given by the low wave number part of the spectrum. The emerging picture of particle-laden turbulence amounts to a standard transfer across the inertial range, a partial removal of energy from the cascade by the Stokes drag and its partial re-injection in the small scales. Under certain respects, the conceptual framework is similar to that operating in polymeric solutions.

The cospectrum, figure 6 , shows a definite alteration of the spectral distribution of the turbulent shear stress and allows to identify the scales affected by the shear stress forcing. Though hardly appreciable in log scale, the cospectrum slightly decreases at large scales as the mass load ratio increases, consistently with the overall reduction of the shear stress. Meanwhile the range affected by production is progressively enlarged. These are the back reaction effects of the highly directional multi-scale clusters which force anisotropic motions in a progressively wider range of scales, see figure 6 . Results at $\Phi=0.2$ present only small deviations from case $\Phi=0$. Increasing the mass load, $\Phi=0.4 \div 0.8$, the back-reaction excites the Reynolds shear stress in almost the entire range of scales. The cospectrum vanishes much more slowly at high wave numbers resulting in unexpected energy injection at small scales. This keeps the turbulent fluctuation excited at scales much smaller than expected on the sole basis of the overall energy
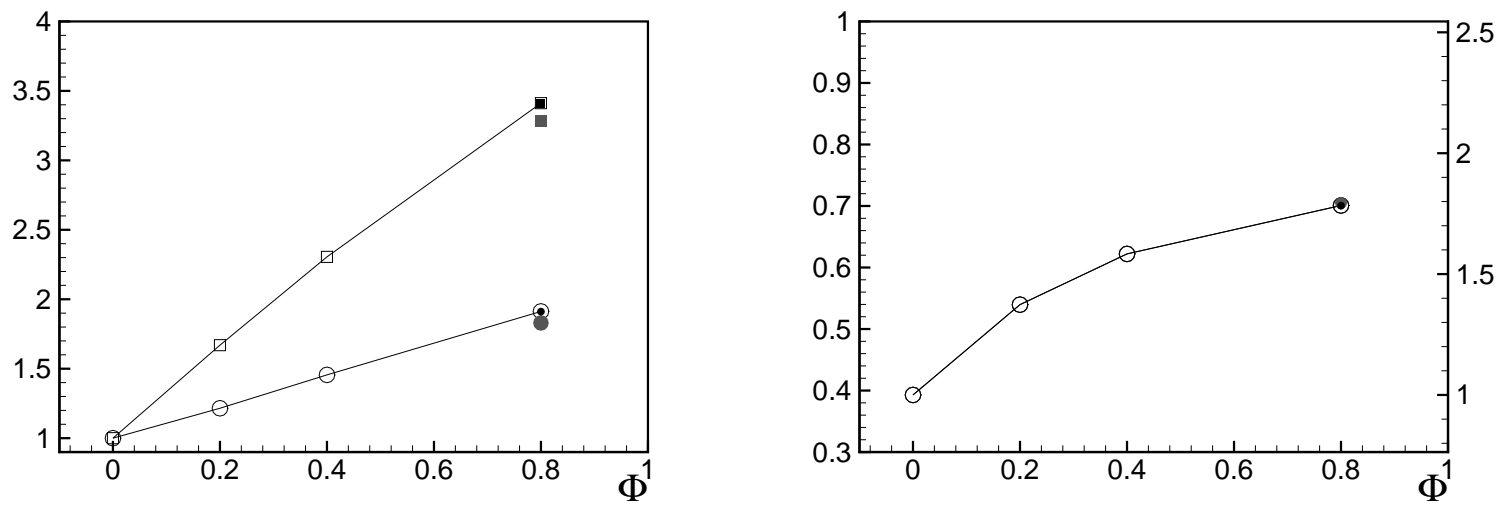

Figure 7. Left: normalized norm of the deviatoric component of the Reynolds stresses tensor $\|b\| /\|b\|_{0}$ (open circles) and of the pseudo dissipation tensor $\|a\| /\|a\|_{0}$ (open squares) as a function of the mass load ratio $\Phi$. For comparison data at $\Phi=0.8$ namely runs $d_{l}$ and $d_{h}$ (grey and black symbols) have been reported. Right: ratio $r=\|a\| /\|b\|$ (circles left axis) and $r / r_{0}$ (circles right axis) as a function of the mass load ratio. Filled symbols as in the left panel. 
production which, as already recalled, diminishes. The insets in figure 6 illustrate the results of grid refinement tests.

The present results apparently question the small scale isotropy assumption for particleladen flows under considerable mass loads, which were classically motivated by the orientational randomization expected of the Richardson cascade.

Concerning the large scales, their anisotropy is quantified by the deviatoric component of the Reynolds stresses tensor $b_{i j}=\left\langle u_{i} u_{j}\right\rangle /\left\langle u_{k} u_{k}\right\rangle-1 / 3 \delta_{i j}$. Since anisotropy is already present at large scales in the Newtonian case (no back-reaction), to isolate the effect of the back-reaction we address, shown by open circles in the left panel of figure 7 , the ratio $\|b\| /\|b\|_{0}$, where the subscript stands for $\Phi=0$ and the norm is $\|b\|=\sqrt{b_{i j} b_{i j}}$. As anticipated in the discussion of figure 5 , the back-reaction enhances the anisotropy. This mainly occurs by the attenuation of the transversal velocity variances $\left\langle u_{2}^{2}\right\rangle$ and $\left\langle u_{3}^{2}\right\rangle$ associated with a slight augmentation of the longitudinal fluctuation $\left\langle u_{1}^{2}\right\rangle$. Concerning the dissipative scales, their anisotropy, shown by open squares in the left panel of figure 7 , is analogously quantified by the deviatoric component of the pseudo-dissipation tensor $\epsilon_{i j}=2 \nu\left\langle\partial_{k} u_{i} \partial_{k} u_{j}\right\rangle$, i.e. by computing $a_{i j}=\epsilon_{i j} / \epsilon_{k k}-1 / 3 \delta_{i j}$ and its norm $\|a\|=\sqrt{a_{i j} a_{i j}}$. The data show a dramatic growth of the gradient anisotropy which under the most severe coupling conditions increases by $350 \%$ with respect to the reference Newtonian case. Two features emerge. First, the disperse phase is able to alter the anisotropy of the largest scale. Second, the small scale anisotropy level normalized with the Newtonian value increases even more steeply with the mass load, due to the spectrally non compact anisotropic forcing operated by the disperse phase on the carrier fluid. Actually the ratio $r=\|a\| /\|b\|$ of small to large scale anisotropy, right panel of figure 7 , almost doubles going from $r=0.4$ for $\Phi=0$ to $r=0.7$ for $\Phi=0.8$, scale on the left axis of the plot.

\section{Final Remarks}

Clustering is a well known feature of inertial particle configurations in turbulent flows. The classical explanation of this small scale behavior is the centrifugal effect due to the vortical structures that populate a turbulent flow, which tend to expel heavy inertial particles from the vortex cores, to localize in the interstices between the vortices. The resulting segregation may be extremely intense for particles at Stokes number order one. A peculiar feature of these systems is their response to the anisotropy of the flow. In recent investigations of a particle-laden homogeneous shear flow it was found, [38], that particle clusters maintain the anisotropy down to their smallest scales, in ranges where, typically, the carrier velocity field already recovered isotropy under the action of the Richardson cascade.

When the mass load ratio is increased, the back-reaction of the suspended phase on the carrier fluid can be no longer neglected, opening the issue of the ultimate fate of the clusters in the strong two-way coupling regime, and poses the question as to how the structure of the turbulence is altered in the carrier phase. This is the regime we have explored in the present paper. As main results, we find that the coupling of the carrier fluid with the suspended phase leads to less pronounced segregation effects, with the clusters that tend to become progressively smeared and less neatly defined with respect to the Newtonian simulation with advected passive inertial particles. At the same time, the anisotropy of the clusters is also reduced. Overall the trend seems to proceed towards a reduction of the peculiar segregation and anisotropy amplification effects recently described for vanishingly small mass loads.

The intriguing aspect is here the response of the carrier fluid to the particle back-reaction. The oriented clusters, though fatter and more isotropic than in the one-way coupling regime, constitute the geometrical support of the force field exerted back on the fluid. This forcing is distributed on the entire range of scales spanned by the clusters and present significant anisotropic features at all active scales. The result is an increased large scale anisotropy of the carrier fluid turbulence, manifested by the Reynolds stress anisotropy indicator. The effect 
consists in the alteration of the energy carrying large scales of the fluid, which is accompanied by a reduction of turbulent kinetic energy. The Reynolds shear stress also diminishes, though less than its isotropic component, resulting in the increase of the anisotropy indicator mentioned above. It follows the reduction in the turbulent kinetic energy production rate, which, at equilibrium, entails a lowering of turbulent energy dissipation rate.

The observation that the back-reaction forcing occurs throughout the range of scales spanned by the clusters, implies that a non-negligible amount of forcing takes place also in the smallest scales of the turbulence, which are usually passively fed only by the energy cascade from larger scales. Consistently, the energy spectrum is broadened, with the excitation of scales significantly smaller than the Kolmogorov scale expected from the energy content of the system. As we have shown, the clusters retain their anisotropy down to the smallest scales. Consistently, the Reynolds shear stress forcing is also much less compact in spectral space than found in classical shear turbulence. This leads to a substantial increase of anisotropy at the level of the instantaneous velocity gradients, as measured by the anisotropy indicator of the pseudodissipation tensor.

In conclusion, the structure of the system of particles and fluid is significantly altered by the coupling, an effect which clearly increases with the amount of mass loading of the suspension. Our findings could have a certain impact on turbulence modeling of multiphase flows which, both in the context of Reynolds averaged (RANS) and filtered equations (LES), heavily rely upon the concepts of inertial energy cascade and of a presumed universal small scale statistics. Actually, we have shown that the cornerstone Kolmogorov theory no longer safely applies, since the classical energy cascade is overwhelmed by the anisotropy-enhancing back-reaction of the particles.

\section{Acknowledgments}

Authors thanks the COST Action MP0806 "Particles in Turbulence" for supporting the present work and the CASPUR consortium where the simulations were performed.

[1] Falkovich G, Fouxon A and Stepanov M 2002 Nature 419151

[2] Wang L, Ayala O, Rosa B and Grabowski W 2008 New J. of Phys. 10 1-40

[3] Károlyi G, Péntek A, Scheuring I, Tél T and Toroczkai Z 2002 Proc. Natl. Acad. Sci. 9713661

[4] Lewis D and Pedley T 2000 J. Thoretical Biology 205 377-408

[5] Woods W A 2010 Ann. Rev. Fluid Mech 42 391-412

[6] Post S and Abraham J 2002 Int. Journal Mult. Flows 28 997-1019

[7] Rouson D and Eaton J 2001 J. Fluid Mech. 428149

[8] Portela L, Cota P and Oliemans R 2002 Powder Technology 125 149-157

[9] Derksen J, Sundaresan S and Akker H V D 2006 Powd. Tech. 163 59-68

[10] Reeks M 1983 J. Aerosol Sci. 14 729-739

[11] Brooke J, Kontomaris K, Hanratty T and McLaughlin J 1992 Phys. Fluids A 6 825-834

[12] Soldati A and Marchioli C 2009 Int. J. Multiphase flow 35(9) 827-839

[13] Marchioli C and Soldati A 2002 J. Fluid. Mech. 468283

[14] Picano F, Sardina G and Casciola C 2009 Phys. Fluids 21093305

[15] Bec J, Biferale L, Cencini M, Lanotte A, Musacchio S and Toschi F 2007 Phys. Rev. Lett. 98(8) 084502

[16] Balachandar S and Eaton J 2010 Ann. Rev. Fluid Mech 42 111-133

[17] Eaton J 2009 Int. J. Multiphase flow 35(9) 792-800

[18] Elghobash S and Truesdell G 1993 Physics of Fluids A: Fluid Dynamics 5(7) 1790-1801

[19] Druzhinin O and Elghobashi S 1999 Physics of Fluids 11(3) 602-610

[20] Druzhinin O 2001 Physics of Fluids 13(12) 3738-3755

[21] Sundaram S and Collins L 1999 J. Fluid Mech. 379 105-143

[22] Ferrante A and Elghobashi S 2003 Physics of Fluids 15(2) 315-329

[23] Boivin M, Simonin O and Squires K 1998 J. Fluid Mech. 375 235-263

[24] Hwang W and Eaton J 2006 Int. J. Multiphase flow 32 1386-1396

[25] Hwang W and Eaton J 2006 J. Fluid Mech. 564 361-393 
[26] Yeo K, Dong S, Climent E and Maxey M 2010 Int. J. Mult. Flow 36(3) 221-233

[27] Bosse T, Kleiser L and Meiburg E 2006 Phys. Fluids 180271021

[28] Yang T and Shy S 2005 J. Fluid Mech. 526 171-216

[29] Geiss S, Dreizler A, Stojanovic Z, Chrigui M, Sadiki A and Janicka J 2004 Exp Fluids 36 344-354

[30] Poelma C, Westerweel J and Ooms G 2007 J. Fluid. Mech. 589315

[31] Poelma C and Ooms G 2006 Appl. Mech. Rev. 50 78-90

[32] Zhao L, Andersson H and Gillisen J 2010 Phys. Fluids 220817021

[33] Yiming L, McLaughlin J, Kontomaris K and Portela L 2001 Phys. Fluids 13(10) 2957-2967

[34] Pan Y and Banerjee S 2001 Phys. Fluids 8(10) 2733-2755

[35] Rani S, Winkler C and Vanka S 2004 Powder Tech. 141 80-99

[36] Ljus C, Johansson B and Almsedt A 2002 Int. J. Multiphase flow 28 1075-1090

[37] Ahmed A and Elghobashi S 2000 Phys Fluids 12(11) 2906-2930

[38] Gualtieri P, Picano F and Casciola C 2009 J. Fluid Mech. 629 25-39

[39] Shotorban B and Balachandar S 2006 Phys. Fluids. 18065105

[40] Casciola C, Gualtieri P, Jacob B and Piva R 2007 Phys. Fluids 19101704

[41] Casciola C, Gualtieri P, Jacob B and Piva R 2005 Phys. Rev. Lett. 95024503

[42] Jacob B, Casciola C, Talamelli A and Alfredsson P 2008 Phys. Fluids 20(4) 045101

[43] Stresing R, Peinke J, Seoud R and Vassilicos J 2010 Physical Review Letters 104194501 ISSN 1079-7114

[44] Rogallo R 1981 Nasa T-M 81315

[45] Maxey M and Riley J 1983 Phys. Fluids 262437

[46] Gualtieri P, Casciola C, Benzi R, Amati G and Piva R 2002 Phys. Fluids 14(2) 583-596

[47] der Hoef M V, Annald M V S, Deen N and Kuipers J 2008 Ann. Rev. Fluid Mech 40 47-70

[48] Burton T and Eaton J 2005 J. Fluid Mech. 545 67-111

[49] Poesio P, Ooms G, Cate A T and Hunt J 2006 J. Fluid Mech. 555 113-130

[50] Zhang Z and Prosperetti A 2005 J. Comp. Phys. 210 292-324

[51] Lomholt S and Maxey M 2003 J. Comp. Phys. 184 381-405

[52] Crowe C, Sharma M and Stock D 1977 J. Fluid Eng. 99325

[53] Garg R, Narayanan C and Subramaniam S 2009 Int. J. Mult. Flow 35 376-388

[54] Gualtieri P, Casciola C M, Benzi R, Amati G and Piva R 2002 Physics of Fluids 14 583-596

[55] Shumacher J 2004 Phys. Fluids 16(8) 3094-3102

[56] Shumacher J, Eckhardt B and Doering C 2010 Phys. Lett. A 374871

[57] Boeck T, Krasnov D and Schumacher J 2010 Physca D 2391258

[58] Grassberger P and Procaccia I 1983 Phys. Rev. Lett. 50346

[59] Arad I, L'vov V and Procaccia I 1999 Phys. Rev. E 596753

[60] Biferale L and Toschi F 2001 Phys. Rev. Lett. 864831

[61] Biferale L, Boffetta G, Celani A, Lanotte A, Toschi F and Vergassola M 2003 Phys. Fluids 15 2105-2112

[62] Chen S, Dhruva B, Kurien S, Sreenivasan K and Taylor M 2005 J. Fluid Mech. 533183

[63] Biferale L and Procaccia I 2005 Phys. Rep. 41443

[64] De Angelis E, Casciola C, Benzi R and Piva R 2005 J. Fluid Mech. 531 1-10

[65] Casciola C and De Angelis E 2007 J. Fluid Mech. 581 419-436

[66] Vaithianathan T, Robert A, Brasseur J and Collins L 2006 J. Non-Newtonian Fluid Mech. 1403

[67] Cai W, Li F and Zhang H 2010 J. Fluid Mech. 665334

[68] Perlekar P, Mitra D and Pandit R 2010 Phys. Rev. E 82066313 